\title{
Phytocercomonas venanatans, a New Species of Cercozoa Associated with Chlorotic Streak of Sugarcane
}

\author{
Chuong N. Ngo, Kathryn S. Braithwaite, $†$ David Bass, Anthony J. Young, and Barry J. Croft
}

First and second authors: Sugar Research Australia, Indooroopilly, QLD, 4068, Australia; third author: Division of Genomics and Microbial Diversity, Department of Life Sciences, Natural History Museum, London, SW7 5BD, United Kingdom and Centre for Environment, Fisheries and Aquaculture Science (Cefas), Barrack Road, The Nothe, Weymouth, Dorset DT4 8UB, United Kingdom; fourth author: Centre for Crop Health, University of Southern Queensland, Toowoomba, QLD, 4350, Australia; and fifth author: Sugar Research Australia, Woodford, QLD 4514, Australia. Accepted for publication 18 December 2017.

\begin{abstract}
Chlorotic streak is a global disease of commercial sugarcane (Saccharum spp. hybrids). The disease is transmitted by wet soil, water, as well as in diseased planting material. Although first recognized almost 90 years ago and despite significant research effort, the identity of the causal agent has been elusive. Metagenomic high throughput sequencing (HTS) facilitated the discovery of novel protistan ribosomal and nuclear genes in chlorotic streakinfected sugarcane. These sequences suggest a possible causal agent belonging to the order Cercomonadida (Rhizaria, phylum Cercozoa). An organism with morphological features similar to cercomonads (=Cercomonadida) was

isolated into pure axenic culture from internal stalk tissues of infected sugarcane. The isolated organism contained DNA sequences identical to those identified in infected plants by HTS. The DNA sequences and the morphology of the organism did not match any known species. Here we present a new genus and species, Phytocercomonas venanatans, which is associated with chlorotic streak of sugarcane. Amplicon sequencing also supports that $P$. venanatans is associated with this disease. This is the first reported member from Cercomonadida showing a probable pathogenic association with higher plants.
\end{abstract}

Chlorotic streak affects commercial sugarcane (Saccharum spp. hybrids) worldwide and was first recognized as a disease concurrently in Java, Hawaii, and Australia around 1929 (Egan 1989; Martin 1935). The disease is characterized by yellow to creamy-white leaf streaks with irregular wavy margins. Necrotic areas within streaks and leaf tips can also be present as well as reddening of the vascular bundles at the nodes within stalks (Martin 1935). Diseased plant crops have reduced height and leaf number, while the subsequent regrowth crop following harvest (ratoon crop) can fail in susceptible cultivars (Braithwaite and Croft 2013; Martin 1935). It is one of the most widespread diseases of the Australian sugar industry and can account for up to $40 \%$ losses in sugar yields per hectare in susceptible varieties (Magarey and Neilsen 2002). It is prevalent in areas of high rainfall, areas prone to waterlogging and disease incidence is especially high in years with above average rainfall (Magarey 2005; Young and Ensbey 2016). Disease is spread through wet soil and by water (drainage, recycled irrigation, and flood) (Magarey and Neilsen 2002; Magarey et al. 2016) and infected planting material, but does not appear to be to spread mechanically (Martin 1935). Disease-free planting material can be attained through a hot water treatment of stalk cuttings at $50^{\circ} \mathrm{C}$ for $30 \mathrm{~min}$ (Egan 1989); however, reinfection can readily occur in the field.

While significant research has been done on the epidemiology and etiology of the disease, identification of the causal agent has been unsuccessful. Previous work has suggested a variety of causal agents with a chytrid initially proposed based on observations of spherical bodies and thalli in stalk sections (Carpenter 1940) and

${ }^{\dagger}$ Corresponding author: K. S. Braithwaite;

E-mail: kbraithwaite@sugarresearch.com.au

Funding: This research was supported by funding from Sugar Research Australia (project 2013357) and the Queensland Department of Agriculture and Fisheries.

*The $\boldsymbol{e}$-Xtra logo stands for "electronic extra" and indicates that one supplementary figure and one supplementary video are published online.

(c) 2018 The American Phytopathological Society roots (Antoine and Ricaud 1966). Abbott and Sass (1945) also observed spherical bodies; however, they believed them to be products derived from the diseased plant. The leafhopper Draeculacephala portola was also implicated as a vector for disease transmission (Abbott and Ingram 1942); however, this was later disproved (Abbott et al. 1961). Disease transmission was definitively shown to be via soil (Antoine 1957) and water (Bird et al. 1958). Transmission experiments by Sturgess (1961) suggested that a virus may be the cause of the disease.

A recent study using generic PCR primers identified oomycete actin primers that produced a PCR product specific to diseased but not healthy sugarcane material. This suggested the casual organism is of eukaryotic origin (Braithwaite and Croft 2013). However, actin genes are often poor phylogenetic markers (Bass et al. 2005) and the actin sequence obtained from chlorotic streak-infected sugarcane did not provide sufficient data for taxonomic classification. The chlorotic streak specific actin sequence had a weak match withplant pathogenic species of Plasmodiophora and Phytophthora. Plant-pathogenic Cercozoa, Oomycota, and Chytridiomycota have characteristics that are consistent with some aspects of the known epidemiology of chlorotic streak of sugarcane such as a motile life stage that spreads through water. We had confidence that high throughput sequencing (HTS) could facilitate the detection of more informative phylogenetic markers to determine the causal agent of chlorotic streak.

The objectives of our study were to (i) use HTS, in particular DNA sequencing (DNAseq), to identify potential plant pathogen DNA sequences associated with chlorotic streak, (ii) determine if organisms identified are associated with the disease using amplicon sequencing, and (iii) formally describe any new species found to be associated with chlorotic streak.

\section{MATERIALS AND METHODS}

HTS. To determine the best tissue for HTS, quantitative PCR (qPCR) targeting the actin diagnostic sequence of Braithwaite and Croft (2013) was performed on xylem exudate, roots, and samples 
along the stalk from various symptomatic field-grown sugarcane plants. DNA was extracted from $100 \mathrm{mg}$ of stalk or root tissue and $2 \mathrm{ml}$ for xylem exudate using a DNeasy Plant Mini Kit (Qiagen, Hilden, Germany). Estimation of pathogen abundance was performed on a ViiA 7 Real-Time PCR System (Applied Biosystems, Foster City, CA) with SensiMix SYBR Low-ROX (Bioline, Alexandria, NSW Australia) using qPCR primers for chlorotic streakassociated actin: qCS Act F1: 5'-GAGCAAGCGTGGTATTCTCA-3'; qCS Act R1a: 5'GAAAAGCAAGGCAACAAACTC-3'; and for sugarcane actin: qSo Act F2: 5' -GTTGCACCACCTGAGAGGA-3'; qSo Act R2: 5'-CATCTGTTGGAAAGTGCTGAG-3'. Primers were used at a $200 \mathrm{nM}$ final concentration, with an annealing temperature of $60^{\circ} \mathrm{C}$. Estimation of relative abundance was performed using the formula 2 (CS actin $\mathrm{Ct}$ - So actin $\mathrm{Ct}$ ).

Samples chosen for HTS library preparation were xylem exudates from diseased and healthy field grown sugarcane cultivar Empire, sourced from Harwood, New South Wales, Australia. Xylem exudate samples were collected under positive pressure (Croft 2002) from stalk pieces after they were thoroughly cleaned with Decon 90 detergent (Decon Laboratories Ltd., East Sussex, UK) and followed by $70 \%$ ethanol. The xylem exudate samples from chlorotic streakinfected plants (CS) were pooled from approximately 35 symptomatic stalks. The control sample $(\mathrm{H})$ was from asymptomatic plants and was pooled from approximately 10 stalks and also included DNA extracted from parenchyma tissue from one asymptomatic stalk. DNA for library construction was extracted using a DNeasy Plant Mini Kit (Qiagen) on a total of 100 and $30 \mathrm{ml}$ of xylem exudate from diseased and healthy plants, respectively. Ten milliliters of xylem exudate was used per extraction and DNA then pooled. Xylem exudate was first centrifuged at $15,000 \times g$ for $1 \mathrm{~min}$ and the pellet used for DNA extraction. Stalk parenchyma tissue from one healthy plant was included to increase the DNA quantity of the $\mathrm{H}$ sample and was extracted as described above. DNA quality and concentration was assessed using both a NanoDrop ND1000 Spectrophotometer and Qubit fluorometer (both Thermo Fisher Scientific, Waltham, MA), and gel electrophoresis.

Libraries were produced using a TruSeq DNA PCR-Free Library Preparation Kit (Illumina, San Diego, CA) that utilizes Covaris shearing to produce a 350-bp median insert size. One hundred base pair paired end sequencing was carried out on one lane of a HiSeq 2000 (Illumina). Library preparation and sequencing were performed by the Ramaciotti Centre for Genomics (University of New South Wales, Sydney, Australia).

Amplicon sequencing. Samples for the two ribosomal amplicon sequencing experiments, were obtained from four cultivars originating from different growing regions (Table 1). Healthy controls were asymptomatic and were from planting material subjected to a hot water treatment of $50^{\circ} \mathrm{C}$ for $30 \mathrm{~min}$. Samples from diseased material were collected from symptomatic stalks. All DNA samples for amplicon sequencing experiments were extracted using a DNeasy Plant Mini Kit (Qiagen). Five field and glasshouse samples in the first experiment (16S, 18S, and ITS amplicon sequencing) were extracted from $9 \mathrm{ml}$ of xylem exudate. The tissue culture plantlet sample was extracted using $100 \mathrm{mg}$ from two small plantlets and DNA then pooled. Tissue culture plants were generated from excised axillary buds from infected plants. Buds were first surface sterilized using a 10-min soak in a bleach solution (sodium hypochlorite $1 \%$ available chlorine wt/vol; 2 drops of Tween 20 per 20$\mathrm{ml}$ solution) and removal of the residual bleach by several washes with sterile water under aseptic conditions. Buds were then treated with tetracycline at $3 \mu \mathrm{g} / \mathrm{ml}$ for $5 \mathrm{~min}$ and grown on Murashige and Skoog (MS) media (Murashige and Skoog 1962) with filter sterilized 6-benzylaminopurine ( $0.2 \mathrm{mg} /$ liter $)$ and kinetin $(0.2 \mathrm{mg} / \mathrm{liter})$ added. Plants were grown in a light room with a $12 \mathrm{~h}$ photoperiod at $27^{\circ} \mathrm{C}$. Plants were confirmed to be positive for chlorotic streak by PCR with the actin diagnostic primer set, actin F1: 5'-GACAACGGCTCCGGT ATGTGCAAG -3'; actin R1: 5'-GTCAGAATCTTCATCATGT AGTCCG-3' (Braithwaite and Croft 2013). A second experiment containing four samples was performed examining only $18 \mathrm{~S}$ amplicons. DNA from the four samples in the second experiment was extracted using between 300 and $600 \mu$ l of xylem exudate.

DNA was provided to the Australian Centre for Ecogenomics (The University of Queensland, Brisbane, Australia) for library preparation and sequencing using the MiSeq platform (Illumina). Primers for prokaryotes were 803F: 5'-TTAGANACCCNNGT AGTC-3'; 1392wR: 5'-ACGGGCGGTGWGTRC-3', which amplifies the small subunit (SSU) 16S ribosomal RNA of bacteria and archaea (16S), specifically the V5 to V8 regions. In Escherichia coli, it amplifies the 803 to 1392 region of the 16 S gene. The fungal primer pair which amplifies the internal transcribed spacer 2 (ITS2) of fungi was ITS3: 5'-GCATCGATGAAGAACGCAGC-3'; ITS4: 5'-TCCTCCGCTTATTRATATGC-3'. In Saccharomyces cerevisiae, it amplifies the entire ITS, including the end of 5.8S and the start of 28S. Other eukaryotes were detected using 563F: 5'-GCCAGCAVC YGCGGTAAY-3'; 1132R: 5'-CCGTCAATTHCTTYAART-3', which amplifies the SSU $18 \mathrm{~S}$ ribosomal RNA of eukaryotes, specifically the V4 and V5 regions. In S. cerevisiae it amplifies the 563 to 1132 region of the $18 \mathrm{~S}$ gene. Reads were discarded if they were single reads, $<0.05 \%$ abundance or less than $60 \%$ sequence identity to the data. Data were only processed on $\mathrm{R} 1$ forward reads to produce a normalized operation taxonomic unit (OTU) table using the Greengenes database (http:// greengenes.lbl.gov) for 16S, the SILVA database (https://www.arb-silva. de/) for $18 \mathrm{~S}$ and the UNITE database (https://unite.ut.ee) for ITS sequences. OTUs were crossed referenced with GenBank and names reassigned where necessary. A cercozoan-Saccharum spp. fusion amplicon was discovered and removed. Several Saccharum spp. and negative control OTUs were also filtered from results for ease of visualization.

Bioinformatics and gene cloning. Bioinformatics analyses were conducted using Geneious version 8 (Biomatters Ltd., New Zealand), unless specified. Sequencing data from the CS sample was used to map to a 1,300 bp actin sequence (Braithwaite and Croft 2013), with default parameters, a 5\% allowable mismatch rate and five iterations used to extend the sequence. Consensus sequences were generated and mapping repeated until no further extension occurred. Several primers were designed to the new putative $5^{\prime}$ and $3^{\prime}$ regions of the sequence using the Genscript online primer tool (https://www.genscript.com/). Specificity was tested with PCR and Sanger sequencing on template DNA from diseased and healthy xylem exudates. Amplification of actin was performed using Phusion High-Fidelity DNA Polymerase (New England Biolabs,

TABLE 1 . Summary of samples examined by community profiling ${ }^{\mathrm{a}}$

\begin{tabular}{llll}
\hline Cultivar & \multicolumn{1}{c}{ Source } & Symptoms & Tissue \\
\hline Q241(b & Field - Tully, QLD & CS & Xylem exudate \\
Empire & Field - Harwood, NSW & CS & Xylem exudate \\
Empire & Tissue culture - Brisbane, QLD & CS & Whole plant \\
RP197-63 & Glasshouse - Woodford, QLD & CS & Xylem exudate \\
RP197-63 & Glasshouse - Woodford, QLD & H stalks & 20 stalks \\
Q155 & Field - Woodford, QLD & CS & Xylem exudate \\
Q155 & Field - Woodford, QLD & H & Xylem exudate \\
Empire & Field - Woodford, QLD & CS & Xylem exudate \\
Empire & Field - Woodford, QLD & H & Xylem exudate \\
\hline
\end{tabular}

${ }^{a}$ First five rows are samples included in 16S, 18S, and ITS amplicon sequencing. Last four rows are samples included in the repeated 18S amplicon sequencing. 
Ipswich, MA) with primers: CS Act F1: 5'-GGTGACTGACGTGG ATTCTTT-3'; CS Act R6: 5' -GCTTCGTTAGTTGCTGATTCAA-3', an annealing temperature of $60^{\circ} \mathrm{C}$, on DNA extracted from cultures by first centrifuging $2 \mathrm{ml}$ of culture at $15,000 \times g$ for $1 \mathrm{~min}$ and extracting cells using a DNeasy Plant Mini Kit (Qiagen). An actin cDNA sequence was generated from infected root RNA extracted using a RNeasy Plant Mini Kit (Qiagen) and cDNA synthesized using Improm II Reverse Transcription System (Promega, Madison, WI). Actin cDNA was amplified with actin $\mathrm{F} 1$ and $\mathrm{R} 1$ primers (Braithwaite and Croft 2013) and sequenced. The cDNA sequence was used to annotate the intron area by aligning to the gDNA sequence.

Initial analysis of HTS data was performed using the Kraken taxonomic sequence classification system (https://ccb.jhu.edu/ software/kraken) on all virus, bacteria, fungi, and protist databases obtained from GenBank. This was followed by analyses using more specific databases based on groups that include plant pathogenic organisms with flagella. Count abundances were compared between diseased and healthy datasets. Saccharum spp. ribosomal sequences (GenBank accession KF184927, 18S position 8033 to 6228 and 28S position 5634 to 2246) were used for mapping analyses. Mapping was performed using default parameters with a $20 \%$ allowable mismatch and three iterations. Alignments were manually examined and novel reads were compared against GenBank sequences using BlastN. Contigs generated from novel reads were aligned back to Saccharum spp. ribosomal sequences. Primers were designed to sequence areas with low homology, PCR verified, Sanger sequenced, and sequences were then used as a reference for further mapping $(0 \%$ allowable mismatch) and sequence extension. This process was repeated until a single unambiguous sequence was achieved. Verification of the $10-\mathrm{kb}$ ribosomal DNA sequence was performed by amplification using LongAmp Taq DNA Polymerase (New England Biolabs) on DNA extracted from cultures and with the primers CS IGS F1: 5'CGTAGAGGAGCAATGCATCA-3'; CS 28S R10: 5'-TGAAACGT TAGTGCCGAGAC-3'. Sequencing was performed using primers designed to cover the length of the sequence. The ribosomal 18S, 5.8S, and $28 \mathrm{~S}$ subunits were annotated by aligning the $18 \mathrm{~S}$ small subunit to Cercomonas plasmodialis (GenBank accession AF411268) and the 5.8S and 28S subunits to Plasmodiophora brassicae (GenBank accession AB526843).

Amplification of polyubiquitin was performed with Phusion High-Fidelity DNA Polymerase (New England Biolabs), using DNA template extracted from cultures and the primers UBIQ1: $5^{\prime}-$ GGCCATGCARATHTTYGTNAARAC-3' and IUB2: 5' -GATGC CYTCYTTRTCYTGDATYTT-3' (Archibald et al. 2003). The UBIQ1 and IUB2 primer pair generates a ladder of ubiquitin gene products corresponding to repeats of polyubiquitin monomers. Fragments between 400 and $900 \mathrm{bp}$ were cloned into pGEM-T Easy Vector Systems (Promega) and sequenced using M13 forward and reverse. The introns were annotated by comparing to a Cercomonas edax (GenBank accession AY099138) amino acid translation.

Culturing. Chlorotic streak infected sugarcane stalks of cultivar RP193-67 from Sugar Research Australia Woodford were first surface cleaned and disinfected with $70 \%$ ethanol. Stalks were split longitudinally under aseptic conditions and approximately 4 to 5 node pieces (approximately $5 \mathrm{~mm}^{3}$ ) were placed into $S 8$ broth (Davis et al. 1980) in Nunc Cell Culture Treated Eas YFlasks (Thermo Fisher Scientific). Cultures were incubated at $28^{\circ} \mathrm{C}$ for 14 days.

Microscopy. Node tissue from infected stalks was fixed with $10 \%$ neutral buffered formaldehyde as well as formalin acetic alcohol and stained with hematoxylin and eosin. Fresh unstained stalk tissue, noderoot squashes and fresh xylem exudate were also examined under a microscope (Olympus BX50, Japan) and Olympus DP controller software was used to capture images. A DM2000 LED microscope (Leica, UK) with phase contrast illumination was also used to capture images and video. Cells growing in S8 were visualized in vivo on an Eclipse E800 light microscope (Nikon, UK). Digital images and measurements were captured using the integrated Leica camera and Nikon LuciaG software.
Phylogenetic tree construction. A cercozoan reference sequence dataset was modified from Howe et al. (2011), including the recently described putative cercomonad Kraken carinae (Dumack et al. 2016). The chlorotic streak associated cercozoan SSU rRNA gene contig $(1,823 \mathrm{bp})$ was added to this and aligned using the E-ins-I algorithm within mafft version 7 (https://mafft.cbrc.jp/alignment/ software/). The resulting alignment (121 sequences, 1781 positions analyzed) was refined manually and analyzed using Maximum Likelihood (ML) in RAxML BlackBox version 8 (http://sco.h-its. org/exelixis/web/software/raxml/) (Generalized time-reversible (GTR) model with CAT approximation (all parameters estimated from the data); bootstrap values mapped onto the tree with the highest likelihood value). A Bayesian consensus tree was constructed using MrBayes v 3.2 .5 (http://nbisweden.github.io/MrBayes/). Two separate $\mathrm{MC}^{3}$ runs with randomly generated starting trees were carried out for 4 million generations each with one cold and three heated chains. The evolutionary model applied included a GTR substitution matrix, a four-category autocorrelated gamma correction and the covarion model. All parameters were estimated from the data. Trees were sampled every 1,000 generations. The first $1 \mathrm{M}$ generations were discarded as burn-in (trees sampled before the likelihood plots reached stationarity) and a consensus tree was constructed from the remaining sample.

\section{RESULTS}

Molecular characterization. Approximately 96 and 99 million 100-bp paired end HiSeq 2000 reads were obtained from CS and $\mathrm{H}$ libraries, respectively. Kraken analyses suggested several candidates based on quantitative differences between the libraries. These included members from the bacterial families Rhizobiaceae, Alcaligenaceae, and Ruminococcaceae, while fungal candidates were from Sclerotiniaceae and Cunninghamellaceae. Many of the eukaryotic sequences were annotated as members of SAR (Stramenopiles, Alveolates, and Rhizaria), including Eupodiscaceae, Fucaceae, Eimeriidae, and Cercomonadidae (n.b. many family level annotations of environmental eukaryotic sequences in GenBank are incorrect).

Mapping the CS data to Saccharum spp. 18S and 28S sequences revealed infrequent reads belonging to Cercozoa. These novel reads were used as seeds to uncover a 1,823-bp cercozoan $18 \mathrm{~S}$ ribosomal sequence (GenBank accession KR704194), followed by a 9,955-bp ribosomal DNA region containing complete ribosomal subunits (GenBank accession MF034900). Remapping of the CS data to the identified ribosomal sequences showed $<1 \%$ of the data belonged to the chlorotic streak associated cercozoan. BlastN showed that the $18 \mathrm{~S}$ nucleotide sequence was $88 \%$ similar to Cercomonas spp., while the $28 \mathrm{~S}$ sequence was $86 \%$ similar to Paracercomonas marina. The $18 \mathrm{~S}$ sequence contains the rare deletion characteristic of Cercozoa (Cavalier-Smith and Chao 2003). Highest similarity of 18 S to the plant pathogenic plasmodiophorids was $81 \%$ to Spongospora subterranea and highest similarity of the $28 \mathrm{~S}$ was $84 \%$ to Plasmodiophora brassicae (n.b. the $28 \mathrm{~S}$ sequence AB526843 of P. brassicae is a chimera, of which the $3^{\prime}$ end corresponds to Neoheteromita globosa, a gliding flagellate related to cercomonads (Howe et al. 2011; Schwelm et al. 2016)).

Phylogenetic analysis of the $18 \mathrm{~S}$ small subunit shows that the chlorotic streak-associated cercozoan branches robustly within the order Cercomonadida (Fig. 1), closely related to an environmental sequence detected in oilseed rape (Brassica napus) rhizosphere samples (OTU558352489), and more weakly related to another environmental sequence from forest soil (FO181520). The most closely related characterized genus was Brevimastigomonas. Concatenated 18S and 28S rDNA phylogenies (Fig. 2) confirm and strengthen this relationship, showing the chlorotic streak associated cercomonad as sister to Brevimastigomonas with maximal Bayesian posterior probability support and moderately strong maximum likelihood bootstrap support (86\%). Based on this phylogenetic distinctiveness, ecology, and morphological characters, we erect a 
new genus and species for the chlorotic streak-associated cercomonad (diagnosis below).

Our study increased the size of the existing diagnostic actin sequence by extension-mapping, PCR, and sequencing. We also identified an associated cercomonad polyubiquitin sequence through PCR with degenerate primers, cloning and sequencing. The actin sequence (GenBank accession MF034898) contains a coding sequence of $1,351 \mathrm{bp}$ and an intron of $208 \mathrm{bp}$. The highest nucleotide similarity of the coding region was $86 \%$ to the insect Helicoverpa armigera, while the highest similarity to other Rhizaria was $82 \%$ to Plasmodiophora brassicae. Mapping of CS and $\mathrm{H}$ data to the actin gene resulted in 50 and 2 reads mapped, respectively. In phylogenetic analyses of this sequence in a dataset including cercomonads, glissomonads, phytomyxids, and other cercozoans, the chlorotic streak actin sequence groups in a clade with Granofilosea and Chlorarachniophyceae (data not shown). As stated previously, the phylogenetic value of actin is poor, however this result at least confirms a filosan cercozoan affiliation, rather than with plasmodiophorids or another other eukaryotic group. For polyubiquitin, several variable monomers were identified and a 904-bp polyubiquitin sequence deposited in GenBank (accession MF034899). The sequence contains the canonical amino acid insertions between the ubiquitin monomers unique to Rhizaria (Archibald et al. 2003).

Cultures, microscopy and taxonomic diagnoses. We isolated an organism consistent with the morphology of Cercomonadida (Bass et al. 2009b) from chlorotic streak-infected plants (Fig. 3A).
Motile cells were first observed after 7 days and, as the population increased, cells appeared to become less active. Ribosomal DNA sequence amplified from contaminant-free cultures was identical to that identified by HTS. Examination of stained and nonstained stalk material and node-root squashes found no evidence of cercomonadlike organisms within cells. However, very small numbers of flagellate cells with morphology consistent with organisms isolated in cultures, were present in xylem exudates expressed from diseased stalks (Fig. 3B) but not from healthy stalks.

Pure cell cultures were used to describe the organism and a new genus has been erected based on differences in molecular and morphological characteristics to the most closely related species of the Cercomonadida, Brevimastigomonas anaerobica. The following description of the organism was made from 2-week-old pure cultures grown on S8 broth.

Genus Phytocercomonas Ngo, Braithwaite, Bass gen. nov. Gliding or swimming cercomonad biflagellates with compact, ovoid bodies, variable in size within type species. Two subapically ventrally inserted flagella. Type species Phytocercomonas venanatans n. sp. Type $18 \mathrm{~S}$ rDNA sequence GenBank accession KR704194. Groups phylogenetically with maximal Bayesian posterior probability support and moderately strong maximum likelihood support as sister to Brevimastigomonas in $18 \mathrm{~S}-28 \mathrm{~S}$ phylogenies.

Etym. A cercomonad associated with plant tissue.

Phytocercomonas venanatans Ngo, Braithwaite, Bass sp. nov.

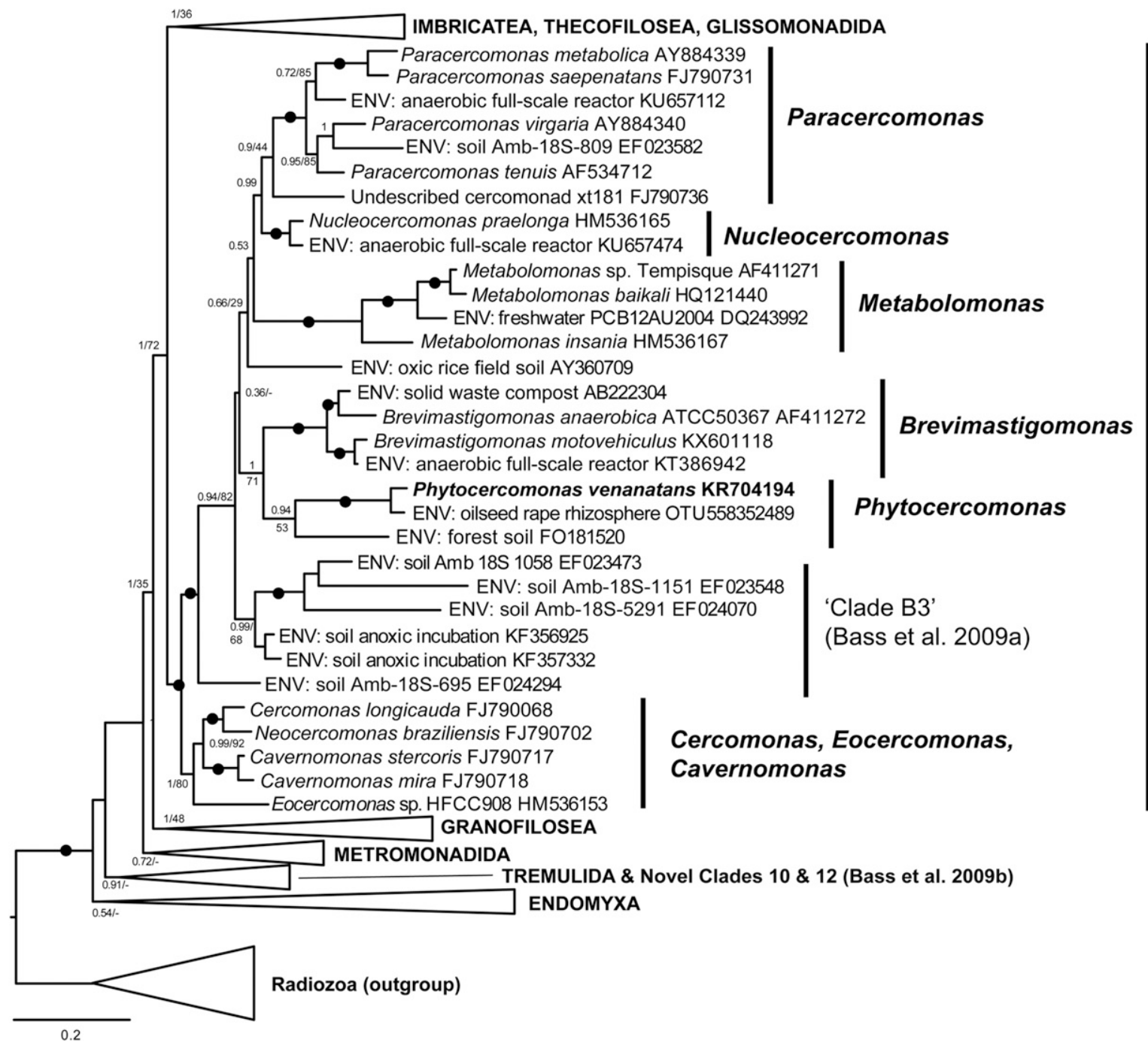

CERCOMONADIDA

Fig. 1. Bayesian 18S rDNA phylogeny including Phytocercomonas venanatans and 110 other cercozoan sequences, rooted on Radiozoa. In this analysis, 1,782 positions were included. Non-cercomonad clades are collapsed for clarity. The fully expanded tree is provided in Supplementary Fig. S1. Bayesian posterior probabilities/maximum likelihood bootstrap values are indicated on nodes; black filled circles indicate values of $\geq 0.95$ and $95 \%$, respectively. 
Type strain CS2_S8, isolated from Saccharum spp. hybrid cultivar RP197-63, Type Figure 3. Type video 1 (Supplementary Video S1).

Diagnosis: Cells round to ovoid to drop shaped, 6 to $15 \mu \mathrm{m}$ long, ventrally flattened. Two flagella, subapically inserted, directed anteriorly (AF) and posteriorly (PF). Flagella extend $+/-$ equally beyond cell in both directions, typically by $1.5 \times$ to $3 \times$ cell length. $\mathrm{PF}$ attached to ventral surface of cell, sometimes in a groove. Both flagella may be (partially) in contact with the substrate when gliding, with most contact, attachment, and motor force provided by PF. Both flagella free and extended when swimming. Gliding movement very variable in speed, from small localized movements to fast (up to $40 \mu \mathrm{m} / \mathrm{s}$ observed) directed gliding, with anteriorposterior rocking/jiggling. Cells frequently not in contact with the substrate, even when not in directed movement. Predivision cells with two anterior and two posterior flagella frequently seen. Swimming motion fast and direct, with flagella extended in front of and behind cell, directing movement by a helical/flickering motion. Cells inflexible in shape; nonmetabolic; small pseudopodia only very rarely observed. One to four contractile vacuoles. Nucleus in a central and anterior position within cell, with prominent nucleolus. No cysts observed, but cells may be stationary in apparent resting mode for extended periods. Nonmarine; grows in organically enriched freshwater culture media. Cryopreservation was unsuccessful utilizing methods for other cercomonads.

Etym. vena $\mathrm{L}$. vein, natans L. swimmer.

Amplicon sequencing. We initially examined prokaryote $16 \mathrm{~S}$, fungal ITS, and eukaryote $18 \mathrm{~S}$ ribosomal sequences in diseased and healthy samples grown under field, tissue culture and glasshouse conditions (Fig. 4A to 4C). We found no bacteria associated with diseased samples (Fig. 4A). The fungal dataset reveals amplification of fungal specific ITS regions as well as off-target amplicons that include bacteria and $P$. venanatans (Fig. 4B). There were no fungi found to be associated with chlorotic streak. Although $P$. venanatans was an off-target result, it contributed between 1.6 to $9 \%$ of total reads from the diseased samples and $<0.001 \%$ in the healthy sample (Fig. 4B). The $18 \mathrm{~S}$ eukaryote data showed $P$. venanatans was present in diseased samples and absent in the healthy sample (Fig. 4C). Eukaryote 18S profiling was repeated using different field grown samples (Fig. 4D). P. venanatans, was again found to be associated with chlorotic streak and contributed 1.3 to $8.6 \%$ of total reads from the diseased and zero in healthy samples. More OTUs are present compared with the initial analysis, due to slight changes in library preparation to deal with low starting quantities of sample DNA.

\section{DISCUSSION}

We have demonstrated the use of new molecular techniques to uncover the elusive and probable causal organism of chlorotic streak of sugarcane. This is the first putative pathogenic association between a cercomonad and a higher plant. The use of HTS to uncover concealed pathogens in both plants (Candresse et al. 2014) and animals (Hartikainen et al. 2014b) is now becoming common place. We used this approach as considerable work has previously been done to identify the causal agent, without success.

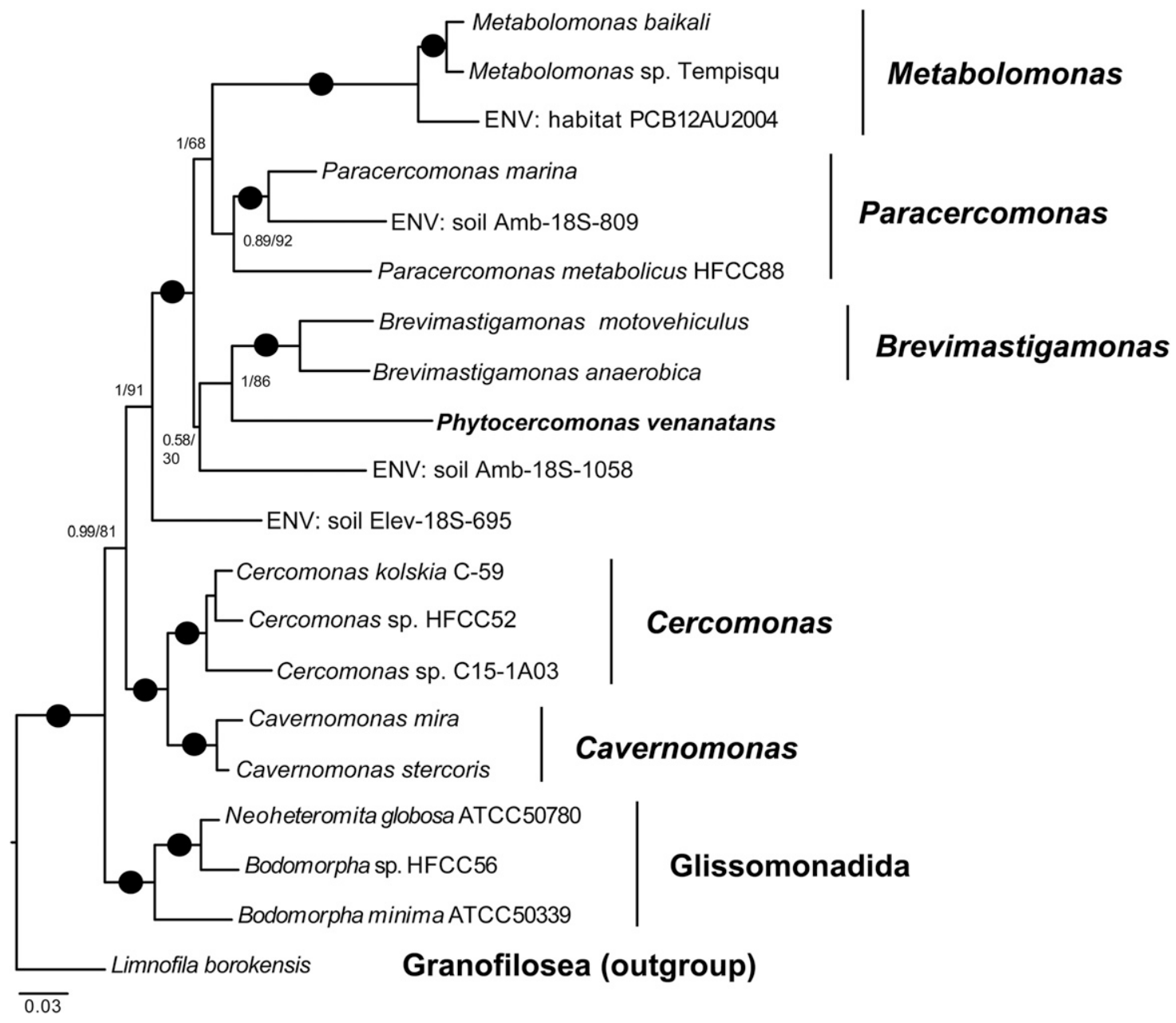

Fig. 2. Bayesian 18S-28S concatenated rDNA phylogeny including Phytocercomonas venanatans and 18 other cercozoan sequences, rooted on the granofilosean Limnofila. In this analysis, 4,556 positions were included. Bayesian posterior probabilities/maximum likelihood bootstrap values are indicated on nodes; black filled circles indicate values of $\geq 0.95$ and $95 \%$, respectively. 
The existing diagnostic actin sequence of Braithwaite and Croft (2013) was especially useful in assisting with selection of pathogenenriched and pathogen-limited samples for DNAseq, allowing for a high chance of successful identification of novel DNA sequences. Quantitative differences between CS and $\mathrm{H}$ datasets using the Kraken program suggested several possible candidates, but this approach was limited due to the novelty of the organism, and therefore the absence of any publicly available DNA sequences. Mapping of the diseased dataset to Saccharum spp. ribosomal sequences revealed a small number $(<1 \%)$ of novel ribosomal reads that were of cercozoan origin. Primers used to create larger ribosomal contigs showed specificity similar to the diagnostic actin sequence (Braithwaite and Croft 2013). The identified ribosomal sequences resulted in an improved diagnostic test with increased sensitivity (Braithwaite et al. 2018).

Initially, the short length of the novel sequences and the absence of closely related sequences on GenBank limited the identification of $P$. venanatans as a cercozoan. Complete ribosomal small and large subunits were assembled revealing similarity to sequences from Cercomonadida. These sequences were identical to ribosomal sequences amplified from $P$. venanatans growing in axenic culture. Further evidence of this affiliation was the presence of a deletion in the 18S small subunit and two amino acid insertions between the polyubiquitin monomers. Rhizaria are unique in having either one or two amino acid insertions between the polyubiquitin monomers, with only Cercozoa containing the deletion in the $18 \mathrm{~S}$ ribosomal sequence (Bass et al. 2005; Burki et al. 2010). P. venanatans has a serineglycine insertion that further differentiates it from the pathogenic Endomyxa group that contain only one polyubiquitin insertion. An intron is also present in polyubiquitin and similar introns are present in several other cercomonads (Archibald et al. 2003).
Amplicon sequencing is used to study often unculturable, microbial communities (Bass et al. 2015; Hartikainen et al. 2014a; Lindahl et al. 2013). Prior to fulfilling Koch's postulates (Braithwaite et al. 2018), we used this technique to determine whether $P$. venanatans is associated with chlorotic streak. Our results indicate a definitive association between the disease and $P$. venanatans with no associations found for bacteria or fungi, suggesting $P$. venanatans is the likely cause of chlorotic streak.

The eukaryotic supergroup Rhizaria comprises of the phylums Cercozoa and Retaria (Foraminifera and Radiozoa) and formal recognition of this supergroup was based on molecular phylogenetics, mainly of the 18S SSU DNA (Burki and Keeling 2014). Rhizaria are one of the most poorly understood supergroups, mainly because many are hard to culture and there are no known rhizarian parasites of humans. The best known pathogenic rhizarians belong to Endomyxa, of which Plasmodiophorida (one of two orders within class Phytomyxea) infect plants and oomycetes (Neuhauser et al. 2014), and members of class Ascetosporea (haplosporidians, paramyxids, paradinids, and mikrocytids) that infect commercially important invertebrates. Plasmodiophorids contain organisms such as Plasmodiophora brassicae, the causal agent of club root in brassicas, Spongospora subterranea, which causes powdery scab of potato, and Polymyxa graminis, an obligate parasite of plant roots, only weakly pathogenic itself, but responsible for transmission of important plant viruses. Haplosporidians (including the genera Haplosporidium, Minchinia, Urosporidium, and Bonamia) and Paramyxida (containing Marteilia, Paramarteilia, Marteilioides, Eomarteilia, and Paramyxa) infect bivalves and crustaceans in both marine and freshwater habitats (Hartikainen et al. 2014a, b; Ward et al. 2016). They can devastate aquaculture and some are listed in international legislation concerning the

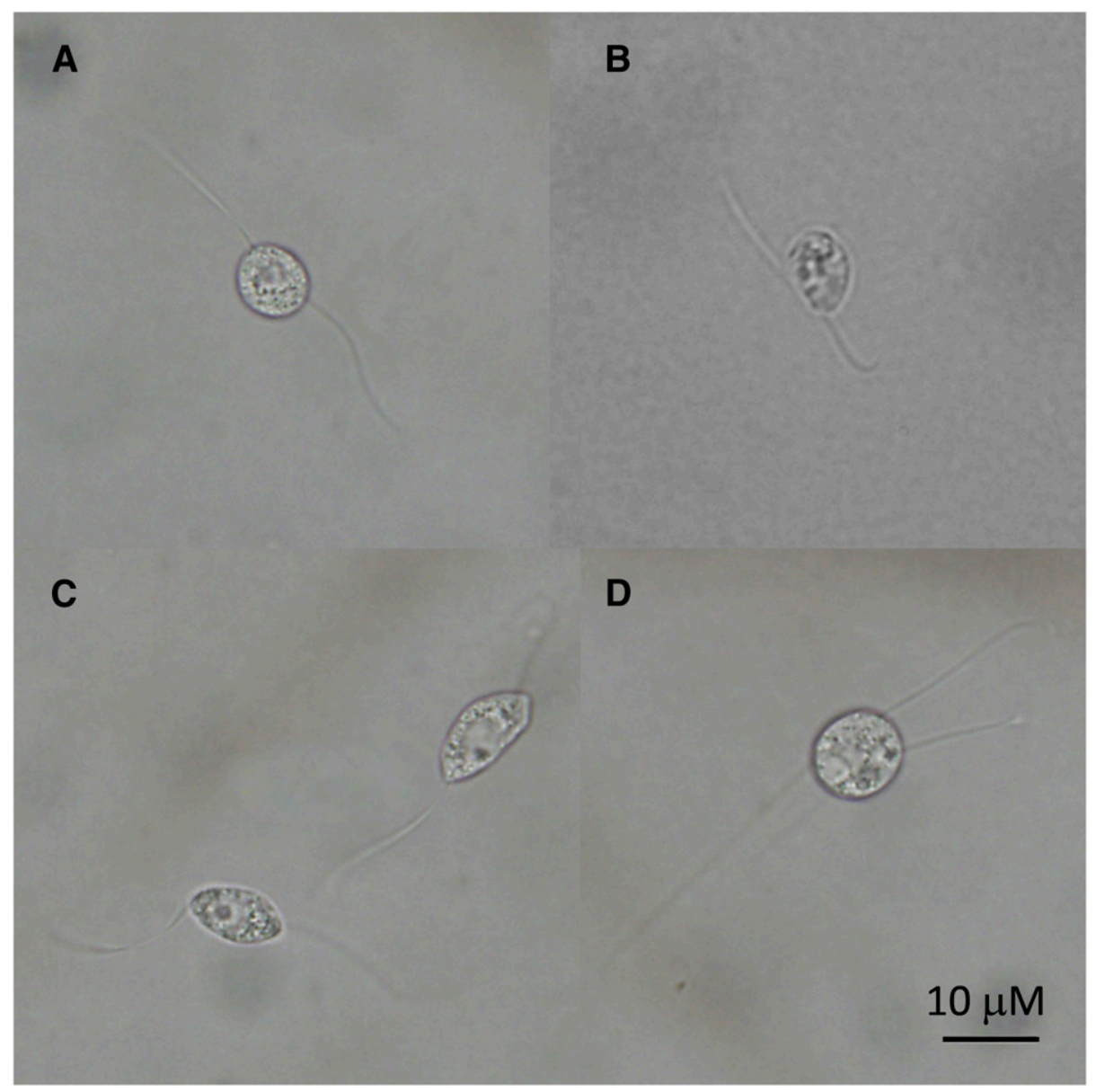

Fig. 3. Phytocercomonas venanatans $\mathbf{A}$, in S8 broth; $\mathbf{B}$, in xylem exudate; $\mathbf{C}$, showing ovoid body form; and $\mathbf{D}$, showing two forward and two reverse flagella. The scale is constant for all figures. 
movement of live animals for aquaculture (Stentiford et al. 2013). However, rhizarian parasites and eukaryvores are not restricted to Endomyxa. Many Cercozoa are flagellates or amoebae, gliding or swimming heterotrophs, and are abundant in marine, soil, and freshwater habitats (Bass and Cavalier-Smith 2004), although the range of cell morphologies is broad and include two lineages that have become algae by enslaving photosynthetic microbes. Cercozoan parasites include Pseudopirsonia and Cryothecomonas, parasites of diatoms, the termite gut endosymbiont Cholamonas, and doubtless many more not yet characterized, but none are yet known from the order Cercomonadida, to which $P$. venanatans belongs.

Cercomonads are particularly abundant in soil and freshwater habitats, where they are important bacterivores, but are also known to predate other microbial eukaryotes and larger organisms, e.g., nematodes (Bjørnlund and Rønn 2008). The diversity of cercomonads has been rapidly expanding in recent years (Bass et al. 2009a;
Brabender et al. 2012; Dumack et al. 2016; Gawryluk et al. 2016). The creation of Phytocercomonas brings the number of genera to eight; prior to Bass et al. (2009b) there was only one. Another putative cercomonad, $K$. carinae (Dumack et al. 2016) was included in a more comprehensively sampled phylogenetic analysis, but groups (albeit very weakly) with imbricates, not cercomonads (Supplementary Fig. S1). This tendency was also noted by Dumack et al. (2016) and its true phylogenetic position awaits clarification. Genomic and transcriptomic analyses of Brevimastigomonas (sister to Phytocercomonas) have recently shed light on the earliest stages of mitochondrial adaptation to low oxygen conditions (Gawryluk et al. 2016); however, Phytocercomonas grows very well in aerobic culture conditions, like most other known cercomonads.

The erection of the new genus Phytocercomonas is supported by the phylogenetic distance from its closest relative Brevimastigomonas, differences in morphology between the two genera and the presence of a related OTU associated with the rhizosphere of oilseed

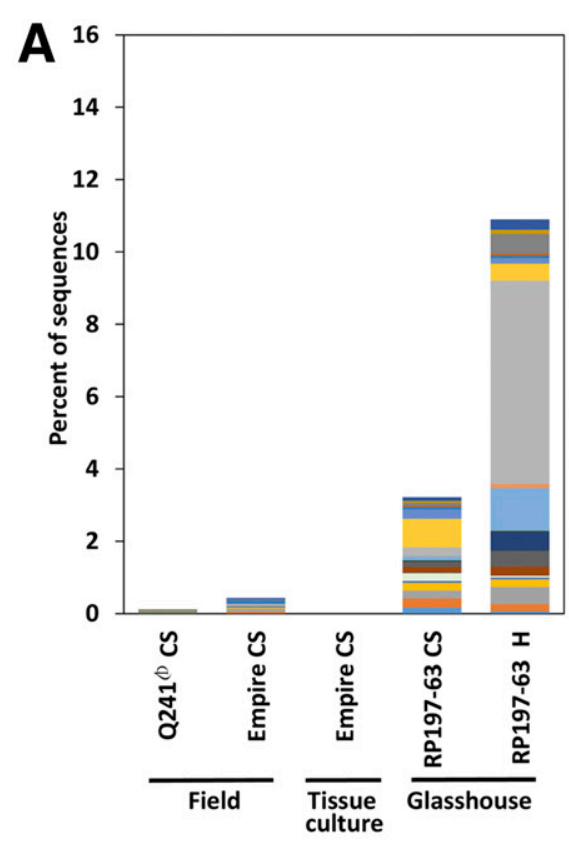

C

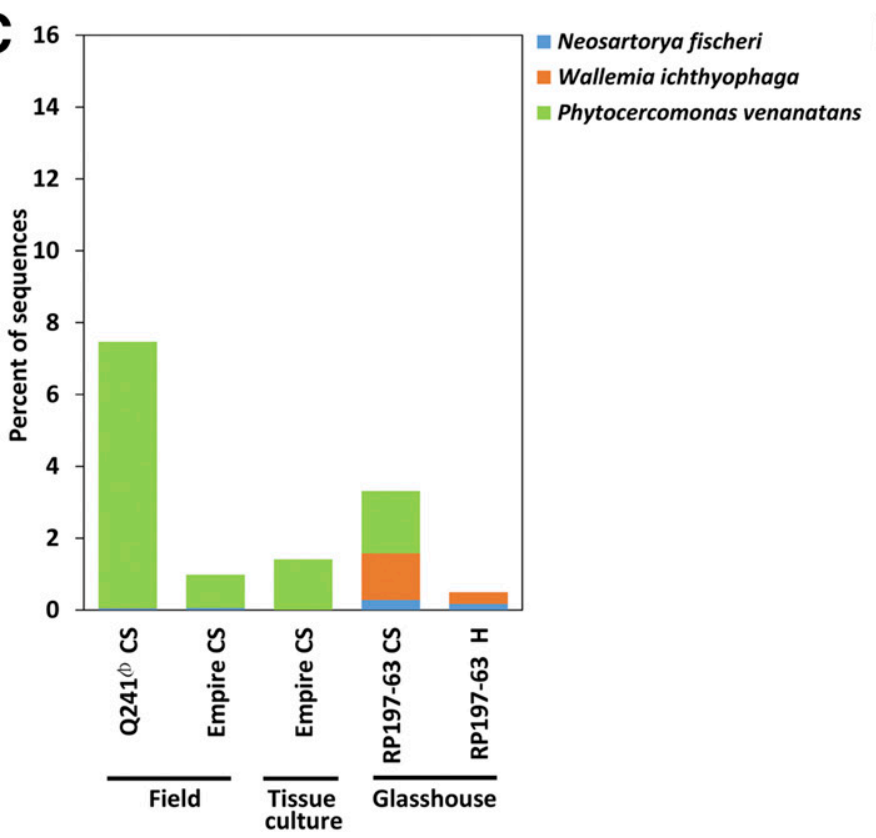

B $^{16}$

Erwinia sp.

Enhydrobacter sp.

- Agrobacterium sp. 2

Sphingomonas yabuuchiae

- Pseudomonadaceae

- Candidatus Tremblaya sp.

n Enterobacteriaceae 1

- Acetobacteraceae

- Xanthomonadaceae 1

- Enterobacteriaceae 2

- Swaminathania sp. 1

= Xanthomonadaceae 2

Swaminathania sp. 2

Pedobacter sp.

Microbacterium chocolatum

- Serratia sp.

- Chryseobacterium sp.

Burkholderia sp.

n Burkholderia gladioli

a Pasteurellales

- Xanthomonadaceae 3
Agrobacterium sp. 1

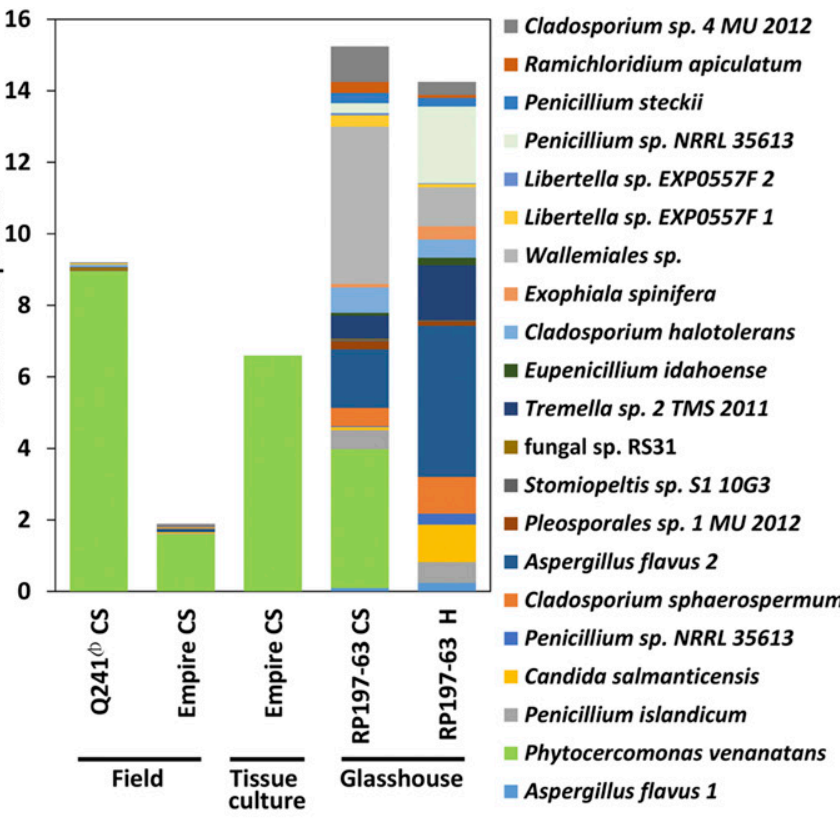

D

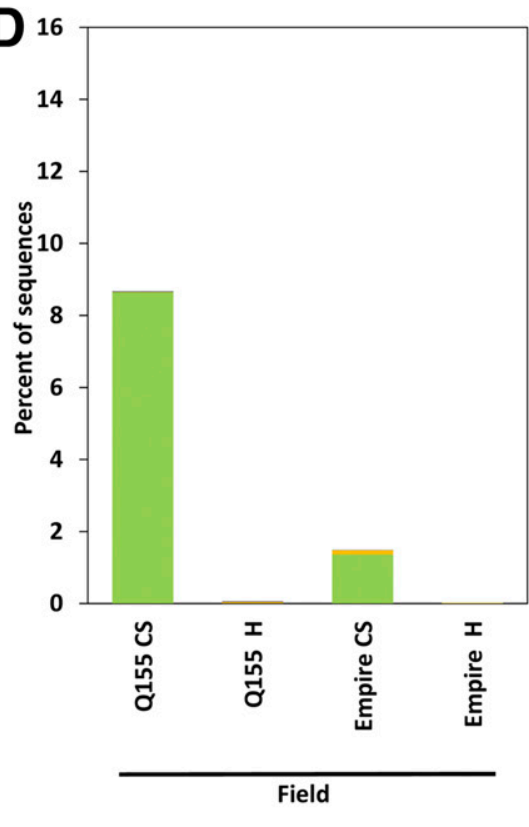

Pseudozyma sp.

Oxalobacteraceae

Phytocercomonas venanatans

Dissoconium aciculare

- Neosartorya fischeri

- Cryptococcus laurentii

- Talaromyces flavus

Bacillaceae

Cryptococcus flavescens

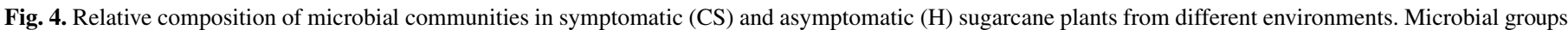

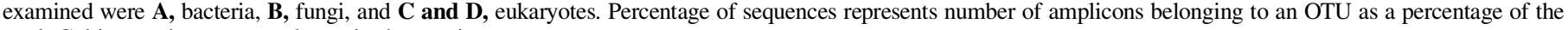
total. Cultivar and source are shown in the $\mathrm{x}$ axis. 
rape (Fig. 1). Morphologically, P. venanatans differs from B. anaerobica in having a larger cell size, longer anterior flagellum, rarely present pseudopodia and it is also nonmetabolic (Bass et al. 2009b; Brabender et al. 2012). Microscopic examination of diseased plant tissues identified $P$. venanatans cells in low counts in xylem exudates, suggesting it is living within xylem vessels. We could not determine if the organism is confined to the xylem or whether it is present in other cells within plants.

Although our study and the fulfillment of Koch's postulates (Braithwaite et al. 2018) are enormous breakthroughs for research on chlorotic streak, much is still unknown about this pathogenic cercomonad and its association with sugarcane. Further work is needed to examine the life cycle of $P$. venanatans within the plant and transmission between plants through soil. Genomic analyses are required to determine whether it shows any physiological adaptations for a plant host-associated lifecycle. A draft genome assembly is currently in progress. It is also unclear whether there is genetic variation present between $P$. venanatans isolates from other countries and whether these organisms were already present in the environment prior to sugarcane farming. The uncovering of this novel plant-pathogenic cercomonad may lead to further discoveries of plant pathogens from this group.

\section{ACKNOWLEDGMENTS}

We thank A. Drenth for discussions on potential pathogens; S. Rudd and QFAB for HTS guidance; R. Magarey for sharing knowledge on chlorotic streak; M. Singh and K. Sventek for assistance with the research; and Tully Cane Productivity Services Limited and Sunshine Sugar staff for providing samples.

\section{LITERATURE CITED}

Abbott, E. V., Hughes, C. G., and Martin, J. P. 1961. Chlorotic streak. Pages 371-387 in: Sugar-Cane Diseases of the World. Vol. I. J. P. Martin, E. V. Abbott, and C. G. Hughes, eds. Elsevier, Amsterdam.

Abbott, E. V., and Ingram, J. W. 1942. Transmission of chlorotic streak of sugar cane by the leafhopper Draeculacephala portola. Phytopathology 32: 99-100.

Abbott, E. V., and Sass, J. E. 1945. Pathological histology of sugar cane affected with chlorotic streak. J. Agric. Res. 70:201-207.

Antoine, R. 1957. Cane diseases 1. Chlorotic streak. Pages 53-57 in: Mauritius Sugar Industry Research Institute Annual Report.

Antoine, R., and Ricaud, C. 1966. Chlorotic streak. Pages 79-80 in: Mauritius Sugar Industry Research Institute Annual Report.

Archibald, J. M., Longet, D., Pawlowski, J., and Keeling, P. J. 2003. A novel polyubiquitin structure in Cercozoa and Foraminifera: Evidence for a new eukaryotic supergroup. Mol. Biol. Evol. 20:62-66.

Bass, D., and Cavalier-Smith, T. 2004. Phylum-specific environmental DNA analysis reveals remarkably high global biodiversity of Cercozoa (Protozoa). Int. J. Syst. Evol. Microbiol. 54:2393-2404.

Bass, D., Chao, E.-Y., Nikolaev, S., Yabuki, A., Ishida, K.-I., Berney, C., Pakzad, U., Wylezich, C., and Cavalier-Smith, T. 2009a. Phylogeny of novel naked filose and reticulose Cercozoa: Granofilosea cl. n. and Proteomyxidea revised. Protist 160:75-109.

Bass, D., Howe, A. T., Mylnikov, A. P., Vickerman, K., Chao, E. E., Edwards Smallbone, J., Snell, J., Cabral, C., Jr., and Cavalier-Smith, T. 2009b. Phylogeny and classification of Cercomonadida (Protozoa,Cercozoa): Cercomonas, Eocercomonas, Paracercomonas, and Cavernomonas gen. nov. Protist 160:483-521.

Bass, D., Moreira, D., López-García, P., Polet, C., Chao, E. E., von der Heyden, S., Pawlowski, J., and Cavalier-Smith, T. 2005. Polyubiquitin Insertions and the phylogeny of Cercozoa and Rhizaria. Protist 156:149-161.

Bass, D., Stentiford, G. D., Littlewood, T. D., and Hartikainen, H. 2015. Diverse applications of environmental DNA methods in parasitology. Trends Parasitol. 31:499-513.

Bird, J., Cibes, H., and Tio, M. A. 1958. Transmission of the causal agent of the chlorotic streak disease of sugar cane through the roots of plants grown in nutrient solution. Univ. Puerto Rico, Agric. Exp. Stn. Tech. Paper No. 27.

Bjørnlund, L., and Rønn, R. 2008. 'David and Goliath' of the soil food webflagellates that kill nematodes. Soil Biol. Biochem. 40:2032-2039.

Brabender, M., Kiss, A. K., Domonell, A., Nitsche, F., and Hartmut, A. 2012. Phylogenetic and morphological diversity of novel soil cercomonad species with a description of two new genera (Nucleocercomonas and Metabolomonas). Protist 163:495-528.
Braithwaite, K. S., and Croft, B. J. 2013. A diagnostic test for chlorotic streak disease. Proc. Austral. Soc. Sugar Cane Technol. 35:8.

Braithwaite, K. S., Ngo, C. N., and Croft, B. J. 2018. Confirmation that the novel Cercozoa Phytocercomonas venanatans is the cause of the disease chlorotic streak in sugarcane. Phytopathology doi.org/10.1094/PHYTO-07-17-0236-R

Burki, F., and Keeling, P. 2014. Rhizaria. Curr. Biol. 24:103-107.

Burki, F., Kudryavtsev, A., Matz, M. V., Aglyamova, G. V., Bulman, S., Fiers, M., Keeling, P. J., and Pawlowski, J. 2010. Evolution of Rhizaria: New insights from phylogenomic analysis of uncultivated protists. BMC Evol. Biol. 10:377.

Candresse, T., Filloux, D., Muhire, B., Julian, C., Galzi, S., Fort, G., Bernardo, P., Daugrois, J.-H., Fernandez, E., Martin, D. P., Varsani, A., and Roumagnac, P. 2014. Appearances can be deceptive: Revealing a hidden viral infection with deep sequencing in a plant quarantine context. PLoS One 9:e102945.

Carpenter, C. W. 1940. A chytrid in relation to chlorotic streak disease of sugarcane. The Hawaiian Planters'. Rec. 44:19-33.

Cavalier-Smith, T., and Chao, E. E. 2003. Phylogeny of Choanozoa, Apusozoa, and other protozoa and early eukaryote megaevolution. J. Mol. Evol. 56: 540-563.

Croft, B. J. 2002. A method for rating sugarcane cultivars for resistance to ratoon stunting disease based on an enzyme-linked immunoassay. Australas. Plant Pathol. 31:63-66.

Davis, M. J., Gillaspie, A. G., Jr., Harris, R. W., and Lawson, R. H. 1980. Ratoon stunting disease of sugarcane: Isolation of the causal bacterium. Science 210:1365-1367.

Dumack, K., Schuster, J., Bass, D., and Bonkowski, M. 2016. A novel lineage of naked filose amoebae; Kraken carinae gen. nov. sp. nov. (Cercozoa) with a remarkable locomotion by disassembly of its cell body. Protist 167:268-278.

Egan, B. T. 1989. Chlorotic streak. Pages 247-262 in: Diseases of Sugarcane Major Diseases. C. Ricaud, B. T. Egan, A. G. Gilaspie Jr., and C. G. Hughes, eds. Elsevier, Amsterdam.

Gawryluk, R. M., Kamikawa, R., Stairs, C. W., Silberman, J. D., Brown, M. W., and Roger, A. J. 2016. The earliest stages of mitochondrial adaptation to low oxygen revealed in a novel rhizarian. Curr. Biol. 26:2729-2738.

Hartikainen, H., Ashford, O. S., Berney, C., Okamura, B., Feist, S. W., Baker-Austin, C., Stentiford, G. D., and Bass, D. 2014a. Lineage-specific molecular probing reveals novel diversity and ecological partitioning of haplosporidians. ISME J. 8:177-186.

Hartikainen, H., Stentiford, G. D., Bateman, K. S., Berney, C., Feist, S. W., Longshaw, M., Okamura, B., Stone, D., Ward, G., Wood, C., and Bass, D. 2014b. Mikrocytids are a broadly distributed and divergent radiation of parasites in aquatic invertebrates. Curr. Biol. 24:807-812.

Howe, A. T., Bass, D., Scoble, J., Lewis, L., Vickerman, K., Arndt, H., and Cavalier-Smith, T. 2011. Novel cultured protists identify deep-branching environmental DNA clades of Cercozoa: New genera Tremula, Micrometopion, Minimassisteria, Nudifila, Peregrinia. Protist 162:332-372.

Lindahl, B. D., Nilsson, R. H., Tedersoo, L., Abarenkov, K., Carlsen, T., Kjøller, R., Kõljalg, U., Pennanen, T., Rosendahl, S., Stenlid, J., and Kauserud, H. 2013. Fungal community analysis by high-throughput sequencing of amplified markers-A user's guide. New Phytol. 199:288-299.

Magarey, R. C. 2005. The incidence of sugarcane diseases in Queensland. Proc. Aust. Soc. Sugar Cane Technol. 27:252-265.

Magarey, R. C., and Neilsen, W. 2002. Chlorotic streak: A disease reducing sugarcane yields in Queensland. Proc. Aust. Soc. Sugar Cane Technol. (CD-ROM) 24:20.

Magarey, R. C., Sventek, K., Bull, J. I., Stringer, J. K., and Zamora, R. 2016. Chlorotic streak resistance screening in the Tully district. Proc. Aust. Soc. Sugar Cane Technol. 38:120-125.

Martin, J. P. 1935. Chlorotic streak disease of sugar cane. Int. Soc. Sugar Cane Technol. Proc. 5:823-828

Murashige, T., and Skoog, F. 1962. A revised medium for rapid growth and bio-assays with tobacco tissue cultures. Physiol. Plant. 15:473-497.

Neuhauser, S., Kirchmair, M., Bulman, S., and Bass, D. 2014. Cross-kingdom host shifts of phytomyxid parasites. BMC Evol. Biol. 14:33.

Schwelm, A., Berney, C., Dixelius, C., Bass, D., and Neuhauser, S. 2016. The large subunit rDNA sequence of Plasmodiophora brassicae does not contain intra-species polymorphism. Protist 167:544-554.

Stentiford, G. D., Feist, S. W., Stone, D. M., Bateman, K. S., and Dunn, A. M. 2013. Microsporidia diverse, dynamic, and emergent pathogens in aquatic systems. Trends Parasitol. 29:567-578.

Sturgess, O. W. 1961. Investigations into chlorotic streak disease. Sugar y Azucar July 1961:29.

Ward, G. M., Bennett, M., Bateman, K., Stentiford, G. D., Kerr, R., Feist, S. W., Williams, S. T., Berney, C., and Bass, D. 2016. A new phylogeny and environmental DNA insight into paramyxids: An increasingly important but enigmatic clade of protistan parasites of marine invertebrates. Int. J. Parasitol. 46:605-619.

Young, A. J., and Ensbey, M. 2016. Insights into the epidemiology of chlorotic streak disease as determined by multiple field assessments. Int. Sugar J. March:216-220. 